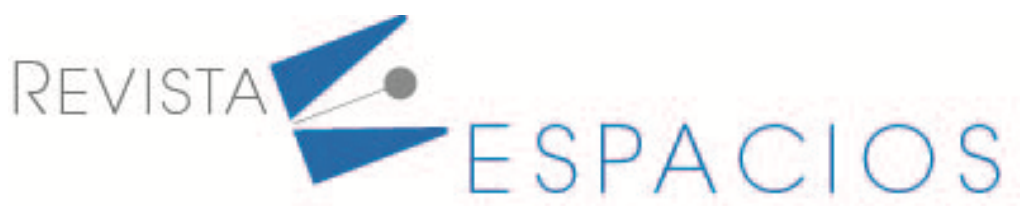

\title{
¿Quo Vadis, televisión comunitaria? Presente y futuro de esta TV de proximidad a partir del análisis de la normativa audiovisual en cuatro periodos presidenciales de Colombia
}

\author{
Quo vadis, community television? Present and future of this proximity TV based on the \\ analysis of audiovisual regulations in four office terms of Colombia
}

\begin{abstract}
ANGULO, Lizandro ${ }^{1}$
Resumen

Esta investigación se centra en el análisis de la legislación comercial y audiovisual que ha reglamentado a la televisión comunitaria de Colombia durante las presidencias de Andrés Pastrana Arango (19982002), Álvaro Uribe Vélez (2002-2010), Juan Manuel Santos Calderón (2010-2018) y la actual de Iván Duque Márquez, para estudiar cómo ha influido esta normativa para que las comunidades organizadas estén abandonando estas experiencias mediáticas y, en ese contexto, cuál es el futuro de esta TV de proximidad. La investigación se realiza mediante un enfoque cualitativo, utiliza el método de la revisión documental y la técnica del análisis textual.

Palabras clave: televisión comunitaria, legislación, gobiernos, Colombia
\end{abstract}

\begin{abstract}
This research goes into analyzing commercial and audiovisual legislation that has regulated these means of communication during the office terms of Andrés Pastrana Arango (1998-2002), Álvaro Uribe Vélez (2002-2010), Juan Manuel Santos Calderón (2010-2018) and the current administration of Ivan Duque (2018-), with the aim of determining the causes of its decline and foreseeing a future scenario. The research is carried out through a qualitative approach, the application of the documentary research, and the textual analysis.

key words: televisión, community, legislation, Colombia.
\end{abstract}

\section{Introducción}

La televisión comunitaria es fundamental para la protección y fortalecimiento de la democracia, puesto que las audiencias pueden participar en la creación, producción y control de los contenidos audiovisuales y en la gestión organizacional para el funcionamiento de este medio de comunicación, en concordancia con la normativa vigente y las necesidades sociales (Angulo, Moreno and Díaz, 2018).

\footnotetext{
${ }^{1}$ Profesor Asociado. Departamento de Ciencias Sociales y Jurídicas. Universidad del Tolima. langulo@ut.edu.co
} 
El modelo colombiano de televisión comunitaria ha sido reconocido internacionalmente por su carácter pluralista, capaz de incentivar la cooperación comunal para la fundación de un medio propio en el que las personas desarrollan competencias organizacionales (administración de un sistema audiovisual) y comunicativas (producir y evaluar programas de TV con una orientación educativa y cultural).

Pese a estas virtudes, estos sistemas han venido desapareciendo del espectro electromagnético nacional en los últimos cinco años, al pasar de 771 en el 2014 a 331 a 24 de julio de 2019, es decir, una reducción del 52\% (Autoridad Nacional de Televisión, 2019). Esta investigación, por tanto, tiene como objetivos identificar cuáles son las razones para que las comunidades organizadas de sus respectivos municipios abandonen estas experiencias mediáticas, analizar si la normativa colombiana puede estar incidiendo en el funcionamiento y sostenimiento de la televisión comunitaria y vislumbrar cuál es el porvenir de esta TV de proximidad en el mediano y largo plazo.

Esta investigación se detiene en el análisis de la legislación comercial y audiovisual que ha reglamentado a este medio de comunicación durante las presidencias de Andrés Pastrana Arango (1998-2002), Álvaro Uribe Vélez (2002-2010), Juan Manuel Santos Calderón (2010-2018) y la actual de Iván Duque Márquez (2018-), con el fin de determinar las causas de su declive y vislumbrar su escenario futuro. Este trabajo se complementa con los resultados del estudio de la Fundación para la Libertad de Prensa (Flip) sobre el estado de la información local, regional y nacional y los Acuerdos de La Habana en los que se subraya el papel de los medios comunitarios en el postconflicto.

\section{Marco teórico}

La TV comunitaria parte de las experiencias latinoamericanas de radio alternativa, particularmente de Radio Sutatenza en Colombia y Radio Mineras Libres de Bolivia, las cuales demostraron que la radiodifusión servía no sólo para informar o hacer propaganda sino también para educar y reivindicar derechos sociales (Gumucio Dagron, 2001).

En los años 60, Canadá se convierte en la nación pionera de la televisión comunitaria, con la transmisión de contenidos que buscaban valorar la identidad cultural de comunidades indígenas de su territorio. A comienzos de los 70, en Estados Unidos, estos sistemas se centraron en elaboración de programas que abogaran por la libre expresión (Chaparro, 2002). En América Latina, las primeras experiencias se presentan en la década de los 80 en Brasil, Colombia y Venezuela con el propósito de promover la identidad cultural y el desarrollo socioeconómico de los pueblos (Noboa Auz, Loor Saltos and Del Pozo, 2018).

El respaldo internacional a estas iniciativas se da en 1976 por el movimiento denominado Nuevo Orden Mundial de la Información y las Comunicaciones (NOMIC), derivado del Informe MacBride, el cual exige la eliminación de los monopolios de comunicación, la descentralización informativa, la difusión democrática de las ideas y la promoción de las capacidades de los países del "Sur" para dotarse de mejores infraestructuras de grabación y transmisión (Angulo, 2012).

Los fundamentos teóricos de la televisión comunitaria difieren de otras modalidades televisivas. Según Dagron (Dagron, 2019), es distinta de la Estatal, toda vez que no constituye el "púlpito" donde los gobiernos se dan propaganda, ni quiere convertirse en un "pulpo" como las cadenas comerciales interesadas por tener más anuncios y rating. Los sistemas comunitarios rescatan el "pálpito" de la comunidad, dando voz a las personas y a grupos de distinta naturaleza. Su incorporación al espectro electromagnético, se debe a la necesidad de "democratizar la palabra en busca de una sociedad más justa, equitativa y solidaria, con la participación de variados actores sociales" (Toro Bravo, Mullo López, \& Hinojosa Becerra, 2019). 
Estos sistemas construyen agendas ciudadanas, y al ser más próximos las comunidades y televidentes hay mayor acierto en lo que la gente siente como de vital importancia para la discusión política. Esta cercanía se traduce en una alfabetización audiovisual, en la medida en que los colectivos aprenden a narrar con la imagen de formas heterogéneas, según sus necesidades, experticia y disponibilidad de recursos económicos y humanos (Parra, 2006).

Las primeras experiencias de TV comunitaria en Colombia surgen en los 80, particularmente con TV Suesca "Nuestra Identidad", a causa de fenómenos del orden tecnológico, legal, educativo y sociológico. En el orden tecnológico, se produce la popularización mundial del satélite para la transmisión de información y la disminución de los costos de los equipos para la producción de video. En el orden sociológico y comunicativo, los canales surgen como (1) espacios para la expresión de las comunidades; (2) en respuesta al desgaste de la televisión estatal que no muestra las realidades de las pequeñas poblaciones; (3) una oportunidad política y económica, para que los dirigentes hagan proselitismo político y los empresarios se lucren con la oferta de canales internacionales a bajo costo y (4) una opción de empleo para los estudiantes de periodismo y comunicación (Rodríguez, 1998). En el orden legal, porque el país afronta procesos de descentralización administrativa que datan de los años 80 y la promulgación de la Constitución Política de 1991, con la que se autoriza a las personas no sólo a recibir información sino a producirla (Artículo 20).

El sostenimiento de la TV comunitaria depende de cada modelo en el mundo. Algunos países como Estados Unidos, Canadá, Brasil y Holanda (Chaparro, 2002; Krohling, 2006) obligan a los operadores por cable (sistema cerrado) a financiar y ceder uno o dos de sus canales a la producción de contenidos comunitarios, pero otros, como el venezolano (Comisión Nacional de Telecomunicaciones de Venezuela, 2002), Chileno (VillarrubiaMartínez, Aguaded-Gómez and Delgado-Ponce, 2019) y belga (Chaparro, 2002), transmiten el canal comunitario de manera abierta, los subvencionan o permiten que les ingresen recursos por donaciones o venta de publicidad.

El modelo colombiano es autosostenible, puesto que una comunidad organizada que crea su medio comunitario en el sistema cerrado puede ofertar a los asociados (televidentes) canales incidentales o gratuitos, codificados (los que pagan derechos de autor) y el canal comunitario por el que se emiten contenidos educativos y culturales. A cambio, el asociado paga mensualmente una cuota mensual o bimestral por el servicio de televisión comunitaria, muy inferior a la que se paga por la televisión por suscripción. También se puede financiar con la venta de publicidad y del servicio de internet (Autoridad Nacional de Televisión, 2018).

\section{Metodología}

Esta investigación se realiza mediante un enfoque cualitativo. Según Jensen (2014), estos estudios se enfocan en el significado, contextos naturalistas y sujetos interpretativos. Este enfoque resulta apropiado para esta investigación, en tanto que lo que se pretende es interpretar cómo la legislación comercial y audiovisual puede estar influyendo en la desaparición y futuro de estos sistemas en el espectro electromagnético de Colombia, regulado por el Ministerio de las TIC y la Comisión de Regulación de las Comunicaciones.

El estudio tiene un diseño narrativo (Hernández, Roberto; Fernández, Carlos y Baptista, 2014), puesto que se contextualiza la época (1998-2018) y el lugar (Colombia) donde aplica la normativa de la TV Comunitaria, así como la secuencia de eventos (periodos presidenciales) en los que se proponen mecanismos para el funcionamiento y sostenimiento de estos sistemas, bajo el amparo de la legislación comercial y audiovisual. La perspectiva elegida de análisis es la histórica, entendida como la investigación que tiene en cuenta los fenómenos comunicativos de la sociedad en el presente y sus antecedentes, con un hacerse histórico, con un antes que los causa y un futuro por venir en etapa de construcción (García, María del Carmen y Berganza, 2005). 


\subsection{Muestras}

Los materiales aquí revisados se constituyen en una muestra diversa (Hernández; Fernández y Baptista, 2014) porque estudia distintas perspectivas y representa la complejidad de un suceso como lo es la transformación de la televisión comunitaria de Colombia desde la presidencia de Andrés Pastrana Arango (1998-2002), Álvaro Uribe Vélez (2002-2010), Juan Manuel Santos Calderón (2010-2018) e Iván Duque Márquez (2018...). Se tienen en cuenta toda la normativa audiovisual de la TV comunitaria de Colombia -salvo los Acuerdos 029 del 19 de diciembre de 1997 y el 006 de 1999 pues sus fundamentos (el origen jurídico de estos sistemas comunitarios en Colombia) se repiten en las normas posteriores-, y la normativa comercial representada por el TLC entre Colombia y Estados Unidos.

\subsection{Analisis de los documentos}

El análisis se lleva a cabo a la normativa audiovisual y comercial. Adicionalmente, se revisa el directorio de TV comunitarias de Colombia para contextualizar el descenso de estos sistemas desde el año 2012 al 2019 y el Informe de la Fundación para la Libertad de prensa en el que se destaca el déficit de información local en Colombia. Estos son:

Tratado de Libre Comercio entre Colombia y Estados Unidos, TLC (Mincomercio de Colombia, 2006), legislación comercial aprobada en el 2007 por el gobierno colombiano de Uribe Vélez y puesta en marcha en el 2012 por el presidente Santos Calderón.

Acuerdo 009 de 2006 de la Autoridad Nacional de Televisión (Autoridad Nacional de Televisión, 2006), bajo el gobierno de Uribe Vélez, por medio del cual se regula el servicio de televisión comunitaria.

Sentencia del Consejo de Estado del 2 de marzo de 2001 y dentro del proceso no 11001-03-24-000-1999-590701 (5907) (Consejo de Estado, 2001), normativa audiovisual, referida a la cobertura de la televisión comunitaria de Colombia.

Resolución 433 de 2013 de la Autoridad Nacional de Televisión (Autoridad Nacional de Televisión, 2013), bajo el mandato de Santos Calderón, por medio del cual se regula el servicio de televisión comunitaria.

Informe del centro de estudios de la Fundación para la Libertad de Prensa del año 2017 (Fundación para la Libertad de Prensa, 2017), sobre el déficit de información local en el territorio colombiano.

Resolución 650 de junio de 2018 de la Autoridad Nacional de Televisión (Autoridad Nacional de Televisión, 2018), bajo el mandato de Santos Calderón, por medio de la cual se regula la televisión comunitaria.

Acuerdo de Paz de La Habana de noviembre de 2016 (Oficina del Alto comisionado para la Paz, 2016), suscrito entre el gobierno colombiano y la guerrilla de las Farc-ep. Normativa sobre postconflicto, en la que se incluye el rol de los medios de comunicación en el proceso. Gobierno de Santos Calderón.

Ley 1978 del 25 de julio de 2019 (Presidencia de la República, 2019), bajo el gobierno de Duque Márquez, por medio de la cual se moderniza el sector de las Tecnologías de la Información y la Comunicación, y el documento El futuro digital es de todos del Ministerio de las Tecnologías de la Información y la Comunicación de Colombia (MinTic Colombia, 2018), en el que se describen los alcances de la Ley 1978 de julio de 2019.

Directorios de la televisión comunitaria, local y por suscripción de Colombia a julio de 2019 (Autoridad Nacional de Televisión, 2019), elaborado por la ANTV. Registro del número de asociados de la TV comunitaria y de suscriptores de la TV por suscripción (ANTV, 2019). 
Gráfico 1

Línea de tiempo de la normativa que ha regido a la TV comunitaria en las presidencias de Pastrana, Uribe, Santos y Duque

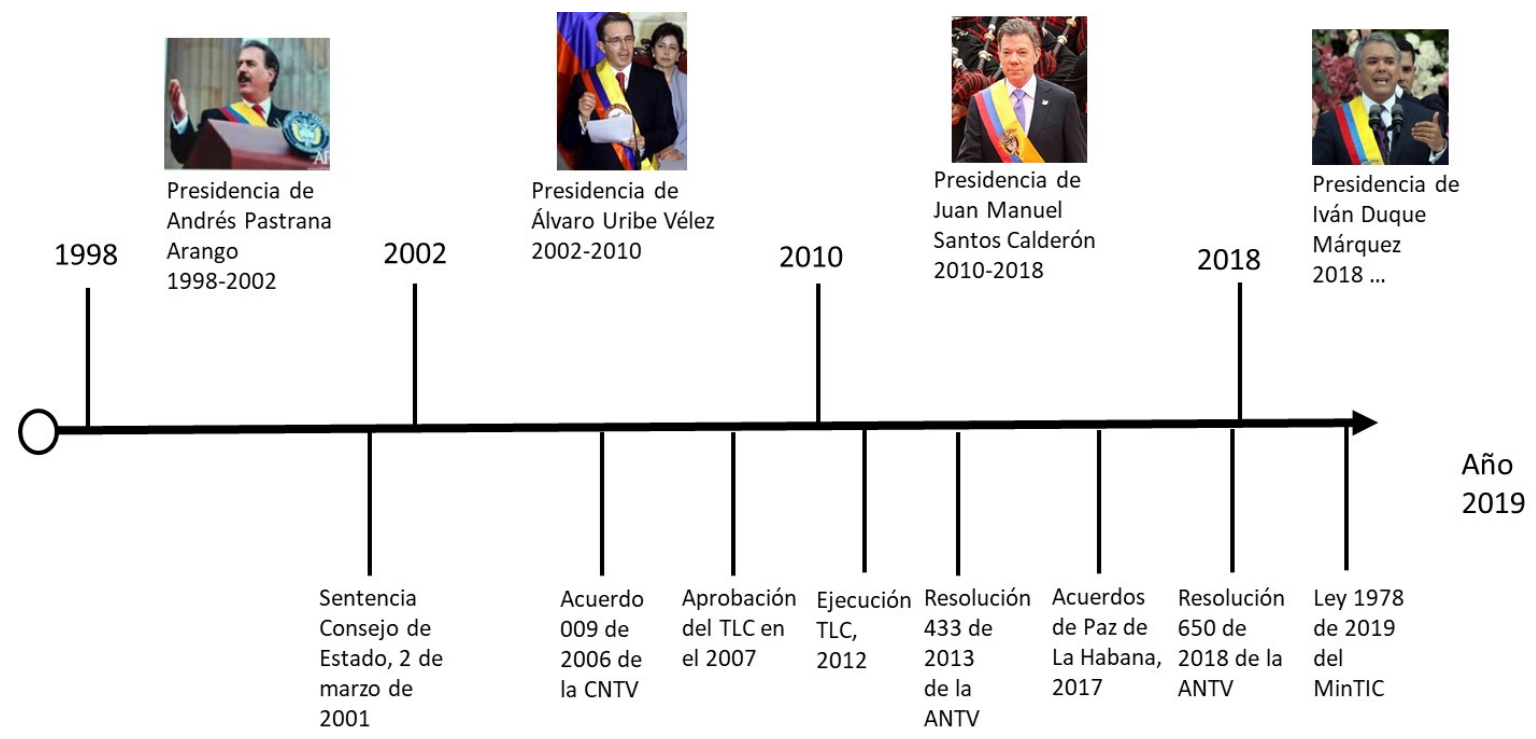

Fuente: elaboración propia

En el gráfico aparecen los Acuerdos, Resoluciones, sentencia del Consejo de Estado y TLC entre Colombia y Estados Unidos (ocho documentos en total), excepto el directorio de las TV Comunitarias de Colombia en tanto que sólo es una relación de estos medios de proximidad que se encuentran vigentes al año 2019.

\subsection{Métodos}

El método empleado es la investigación documental, el cual consiste en la revisión de información cuantitativa y cualitativa de manera cuidadosa y sistemática, a fin de contextualizar un fenómeno y estar al día con lo que circula en torno al tema que se estudia (Toro y Parra, 2010). Usualmente, para la labor de revision de estos documentos cualitativos se requiere del concurso de otra técnica como el análisis interpretativo que, de acuerdo con Neuendorf (2002), desarrolla explicaciones de cómo se produce un fenómeno. En particular, de cómo se puede estar produciendo el descenso de las TV comunitarias de Colombia por la influencia de su normativa comercial y audiovisual.

Los datos se analizan con el método hermenéutico, con el fin de comprender el espíritu de la legislación audiovisual y comercial de la TV comunitaria, la incidencia en su funcionamiento y sostenimiento en el marco de cuatro periodos presidenciales de Colombia, de modo que vislumbre el futuro de estos sistemas en el ecosistema mediático del país.

\subsection{Procedimientos}

La investigación se llevó a cabo bajo el cumplimiento de estas etapas:

Revisión de documentos sobre televisión comunitaria, especialmente los modelos más conocidos en el mundo y sus fundamentos teóricos.

Estructura del marco teórico a partir de categorías de análisis extraídas con el apoyo del software Nvivo para el análisis de información cualitativa. 
Selección de los documentos para el análisis textual, en función de la normativa audiovisual y comercial que reglamenta el servicio cerrado de televisión comunitaria de Colombia. Se incluyen el informe de la Fundación para la Libertad de Prensa sobre el déficit de información local en el país y el directorio de TV comunitarias desde el año 2012 a 2019. Uso del programa Nvivo.

Interpretación y cruce de la información para el cumplimiento de los objetivos de investigación.

\section{Resultados}

\subsection{La televisión comunitaria en la presidencia de Andrés Pastrana Arango (1998-2002)}

La Sentencia del Consejo de Estado del 2 de marzo de 2001 expedida durante la presidencia de Pastrana, limita a 6.000 asociados la cantidad de asociados de la televisión comunitaria. La norma aduce que el ámbito geográfico es importante para (1) evitar "prácticas monopolísticas en el uso del espectro electromagnético" como lo advierten los artículos 75 y 76 de la Constitución Nacional y (2) proteger el propósito de la televisión comunitaria, esto es, "alcanzar fines cívicos, recreativos, culturales o institucionales", porque, de no hacerlo, este servicio "podría extenderse a otros sectores que no tienen las mismas necesidades y objetivos, desnaturalizándose la finalidad del mismo" (Consejo de Estado, 2001).

Tal sentencia justifica la reducción del número de asociados a sólo 6.000 (antes de ella se permitían hasta 15 mil asociados), para supuestamente evitar que las televisiones comunitarias se vuelvan "monopolios" audiovisuales en las grandes y pequeñas ciudades, cuando éstas tienen que competir con los grandes conglomerados de los medios por suscripción que cuentan con más recursos humanos, económicos, financieros y tecnológicos.

\subsection{La televisión comunitaria en la presidencia de Álvaro Uribe Vélez (2002-2010)}

La expedición del Acuerdo 09 de 2006 de la Comisión Nacional de Televisión coincide con la presidencia de Uribe Vélez. La norma crea las bases para que la televisión comunitaria pueda funcionar y sostenerse con los dineros pagados por los asociados y por la pauta comercial. De hecho, en el año 2007 el número de licencias activas fue de 714, distribuidas por todo el territorio nacional, lo que significa un avance en el desarrollo de empresas de medios comunitarios en el país. Ello fue posible porque establece disposiciones claras en términos de cobertura, distribución de canales codificados, pagos por compensación a la CNTV para subsidiar la televisión pública de Colombia, producción propia de contenidos y otorgamiento de licencia única. La Comisión Nacional de Televisión fue el primer organismo autónomo, encargado de regular el servicio de televisión en el país. Se eliminó en el 2012 para dar paso a la Autoridad Nacional de Televisión que estuvo vigente hasta el 2019, cuando el gobierno de Duque decide liquidarla y transferir parte de sus funciones a la Comisión de Regulación de las Comunicaciones.

En cobertura, el operador comunitario puede tener, como máximo, 15 mil asociados, pese a que la Sentencia del Consejo de Estado la reduce a 6.000. No obstante, la antigua Comisión Nacional de Televisión aprueba el tope de 15.000 si es para cumplir fines sociales, comunitarios o de interés público (Artículo 11). En distribución de canales codificados, la comunidad organizada puede ofertar hasta siete (7) señales codificadas, es decir, las que pagan derechos de autor, siempre que cumpla con el tiempo mínimo de producción propia (Artículo 7). Asimismo, podrán recibir señales incidentales porque son libres, toda vez que se destinen al disfrute privado y a fines sociales y comunitarios (punto 4 del Artículo 6). En pagos por compensación, la televisión comunitaria paga el 7\% de los ingresos brutos mensuales para financiar la TV pública del país, incluidos los aportes de los asociados, en razón de un $1 \%$ por cada canal codificado que emita. Si no emiten canales codificados, en todo caso deben pagar el 1\% de los aportes de los asociados (Artículo 14). En contenidos propios (programación), las comunidades organizadas con una cobertura reportada a la CNTV inferior o igual a 2.000 asociados, deben producir 1 hora semanal; las que tengan una cobertura mayor a 2.000 asociados y hasta 8.000 asociados, 2 horas semanales de 
producción propia y media hora semanal adicional por cada señal codificada que emita y las de una cobertura mayor a 8.000 usuarios, 2 horas semanales de producción propia, una hora adicional por cada mil usuarios y media hora semanal adicional por cada señal codificada que emita, hasta un total de 10,5 horas semanales (Artículo 17). En otorgamiento de la licencia, la CNTV prevé un permiso de operación único indefinido, es decir, una concesión nunca caducable.

Estas condiciones, tropiezan con la aprobación del Tratado de Libre Comercio en el 2007 entre Colombia y Estados Unidos, pues el documento que establece la alianza comercial contempla que:

Para mayor certeza, estos servicios presentan restricciones respecto al área de cubrimiento, número y tipo de canales; pueden ser ofrecidos a no más de 6.000 asociados o miembros comunitarios; y deben ser ofrecidos bajo la modalidad de canales de acceso local de redes cerrados (Adenda I,I-COL-25 del TLC) (Mincomercio de Colombia, 2006).

Es decir, el TLC adopta lo prescrito por la Sentencia del Consejo de Estado en la que pone tope a los asociados (6.000) para supuestamente evitar prácticas monopolísticas, y que los fines de la televisión comunitaria choquen contra la de otros sectores poblaciones. Cabe aclarar que el TLC empieza a ejecutarse cinco años después, esto es, en el 2012.

\subsection{La televisión comunitaria en la presidencia de Juan Manuel Santos Calderón (2010- 2018)}

La televisión comunitaria en la presidencia de Santos Calderón está marcada por la aplicación del Tratado de Libre Comercio (TLC) entre Colombia y Estados Unidos y la Resolución 433 de 2013, normas que inician el declive de los sistemas comunitarios en todo el país. En efecto, en cobertura, el operador comunitario sólo puede tener como máximo 6.000 asociados (Artículo 10) para dar cumplimiento a lo pactado en el TLC. Es decir, le arrebata 9000 asociados puesto que el Acuerdo 009 de 2006 permitía el ingreso de 15.000. Pero no es la única disposición que afecta su funcionamiento y sostenimiento.

En la distribución de señales codificadas, se mantiene el número de siete (7), Sin embargo, ese número se puede ampliar sólo si son canales temáticos nacionales que produzcan y transmitan contenidos desde el territorio nacional, con previa autorización de la ANTV (Artículo 15). Así las cosas, no se pueden ofertar más canales codificados internacionales, de modo que el operador comunitario pueda competir con la oferta ilimitada de la televisión por suscripción.

En pagos por compensación, sin duda que asesta un duro golpe a la financiación de los sistemas comunitarios, ya que deben pagar a la ANTV una compensación por la explotación del servicio público de televisión, la cual será el resultado de multiplicar el número total de asociados al mes por el Valor de Compensación por Asociado al Mes. Para determinar el Valor de Compensación por Asociado, los licenciatarios de TV comunitaria deben ubicar el rango de Necesidades Básicas Insatisfechas (NBI) en el que se encuentra el municipio (Artículo 12). En otras palabras, las comunidades organizadas pagarán más a la ANTV que cuando lo hacían a la desparecida CNTV mediante el abono del $1 \%$ por canal codificado emitido.

En contenidos propios (programación), deben producir mínimo cinco (5) horas de programación propia semanal desde el inicio de operaciones y hasta un (1) año después. A partir del segundo año de operación de los nuevos licenciatarios, y para los licenciatarios habilitados antes de la entrada en vigencia de la presente resolución, se debe aumentar la producción propia en mínimo cinco (5) horas de producción propia de lunes a viernes, y dos (2) horas de producción propia los sábados y los domingos, con el fin de completar un mínimo de nueve (9) horas semanales. La coproducción sólo puede alcanzar hasta el 50\% de la programación y es obligatorio destinar dos 
horas para la producción independiente de sus asociados (Artículo 16). El error de esta decisión estriba en que estandariza la producción propia, sin tener en cuenta el número de habitantes de un municipio. No se puede pedir cinco horas de producción semanal a un municipio de 3000 habitantes que a uno de 150.000, pues este último cuenta con más recursos humanos, financieros, logísticos, así como con más actividad política, económica y cultural.

Como se deduce, la vigencia de la Resolución 433 de 2013 tuvo como consecuencia el declive cuantitativo de la TV comunitaria en el país. De igual manera, se expone el comportamiento de las televisiones locales sin ánimo de lucro y por suscripción (gráfico 2).

\section{Gráfico 2}

Declive de las televisiones comunitarias de Colombia

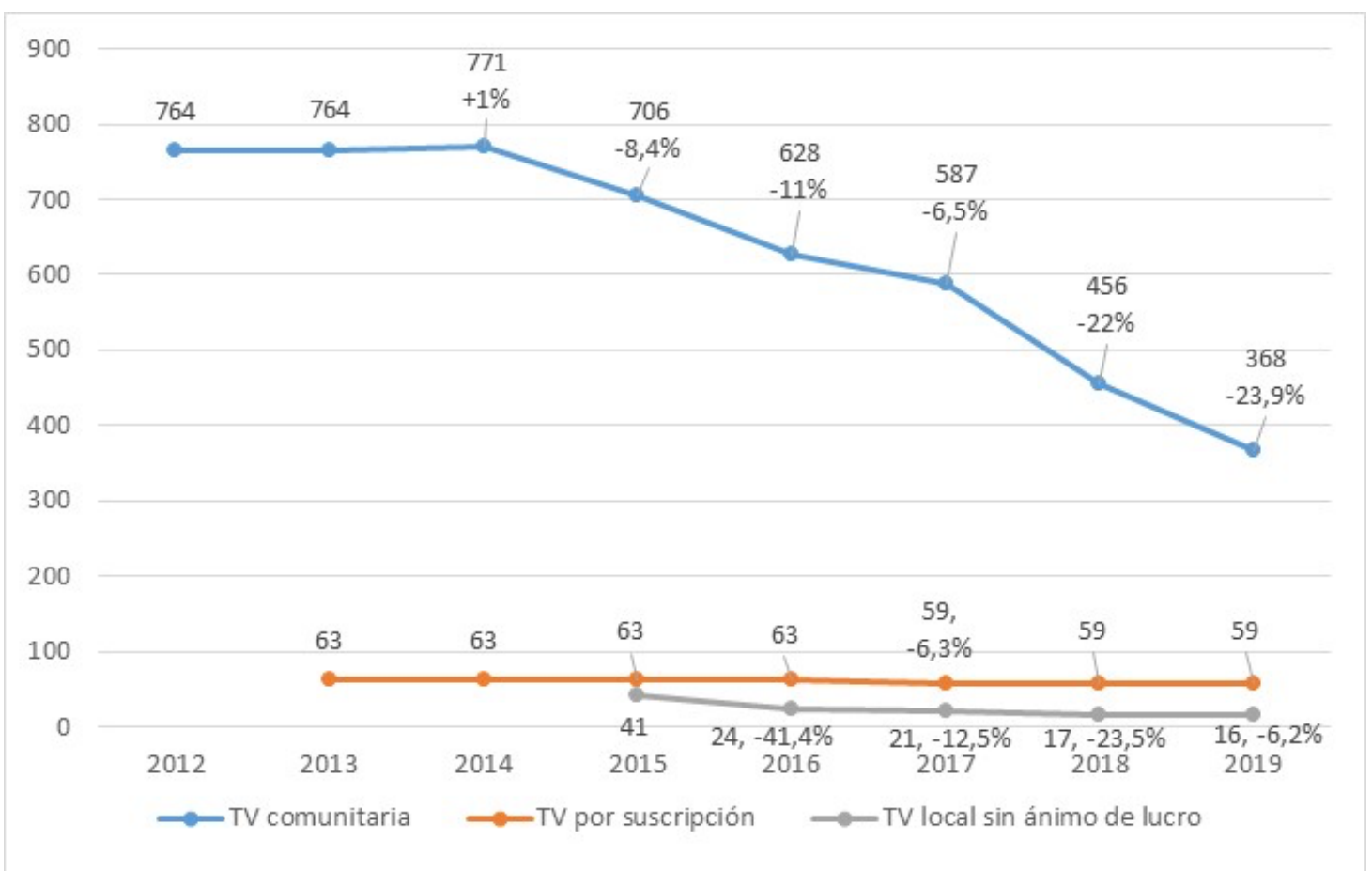

Fuente: elaboración propia a partir de los reportes de la ANTV

En efecto, entre los años 2014 y 2015 el número de sistemas comunitarios empieza a disminuir en un 8,4\%. De ahí en adelante, la reducción es vertiginosa hasta el punto de que en el periodo comprendido entre 2014 y a julio de 2019 el acumulado es del 52\%, es decir, un poco más de la mitad de estas televisiones ha desparecido. Una circunstancia parecida a la de los sistemas comunitarios acontece con la TV local sin ánimo de lucro, perteneciente a la modalidad de señal abierta, la cual ha venido disminuyendo al pasar de 41 en el 2015 a 16 en el 2019. En otras palabras, el $60 \%$ ya no existe en el espectro electromagnético del Estado. Mientras eso ocurre, el número de televisiones por suscripción se ha mantenido prácticamente estable, puesto que sólo cuatro de ellas han dejado de funcionar entre los años 2016 y 2017.

Otro dato relevante es el referente al crecimiento sostenido de la cantidad de suscriptores de la televisión por suscripción desde el año 2012 hasta mayo de 2019, mientras que los asociados de la televisión comunitaria van en franco descenso en el mismo periodo, debido a la normativa audiovisual comunitaria, especialmente la Resolución 433 la cual ha provocado un decrecimiento de operadores y, en consecuencia, una disminución acumulada del 54,3\% número de asociados en los siete años analizados (ver ilustración). Es probable que muchos 
de ellos hayan sido captados por los operadores por suscripción, los cuales han visto que en los mismos siete años el crecimiento acumulado de suscriptores ha sido del $37,8 \%$.

Gráfico 3

Número de asociados de la TV comunitarias y número de suscriptores de la TV por suscripción

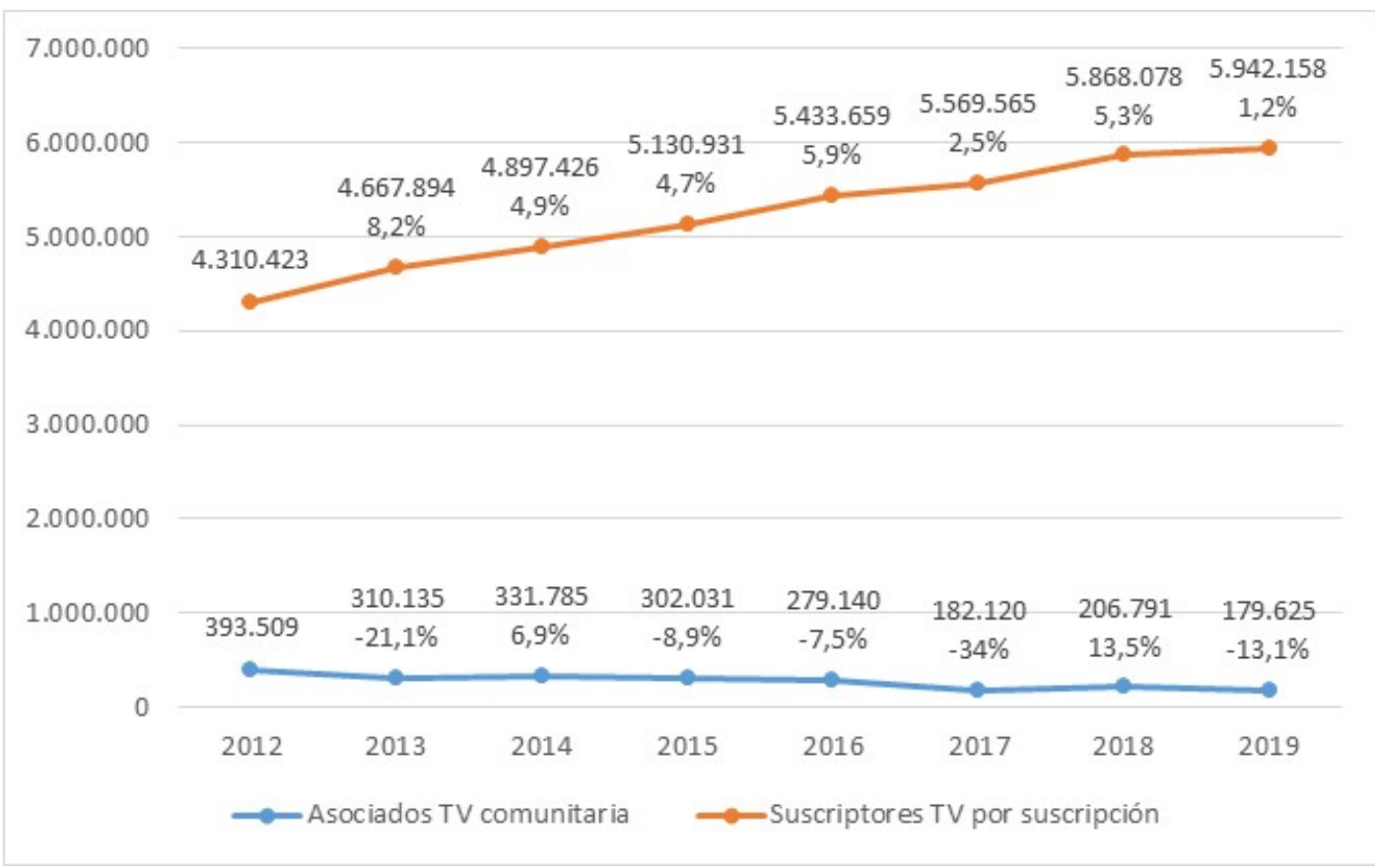

Fuente: elaboración propia a partir de los reportes de la ANTV

El déficit de información local diagnosticado por el Centro de Investigación de la Fundación para la Libertad de Prensa (Flip) en el 2017, puede estar vinculado con la eliminación de algunas televisiones comunitarias y locales en 23 departamentos de Colombia (tabla 1). Este fenómeno se entiende como la ausencia de informción o de medios de comunicación locales, lo cual atenta contra la cultura democrática porque los ciudadanos no tienen la posibilidad de conocer su entorno más cercano ni de tener un canal para expresarse libremente sobre la vida política, cultural y económica de su lugar de residencia.

\section{Cuadro 2}

Déficit de información local por departamento de Colombia

\begin{tabular}{|c|c|c|c|c|}
\hline $\begin{array}{c}\text { Departamentos } \\
\text { estudiados }\end{array}$ & $\begin{array}{c}\text { No de habitantes por } \\
\text { los municipios } \\
\text { estudiados }\end{array}$ & $\begin{array}{c}\text { № de municipios } \\
\text { estudiados }\end{array}$ & $\begin{array}{c}\text { Municipios sin } \\
\text { información local }\end{array}$ & $\begin{array}{c}\% \text { del total de } \\
\text { municipios sin } \\
\text { información local }\end{array}$ \\
\hline 23 & 28.086 .234 & 690 & 392 & $56,8 \%$ \\
\hline
\end{tabular}

Fuente: la Flip (Fundación para la Libertad de Prensa, 2017)

El resultado contundente del informe es que, de los 690 municipios mapeados, 392 (el 56,8\% de ellos) no disponen de medios de comunicación locales o próximos o, si hay algunos, no producen información local.

Con la Resolución 650 de 2018, expedida al final del gobierno Santos, se quiere mejorar las condiciones económicas para el funcionamiento y sostenimiento de las televisiones comunitarias del país. La disposición legal mantiene la cobertura autorizada, esto es, 6.000 asociados como máximo en concordancia con lo señalado por el TLC con Estados Unidos, sin embargo, dicha cantidad puede aumentar cuando el licenciatario comunitario 
informe a la ANTV y remita copia del plano esquemático, sin que pueda superar los límites geográficos del respectivo municipio (Parágrafo 2 del Artículo 14). Lo que no precisa es a cuántos nuevos asociados puede aspirar.

Otra modificación es sobre el valor de compensación. En la Resolución 433 de 2013 estipula que el operador comunitario debe pagar a la ANTV un porcentaje por cada asociado, en tanto que la Resolución 650 manifiesta que este valor se calculará de acuerdo con los ingresos brutos mensuales provenientes de la explotación del servicio de TV y según la cantidad de habitantes del municipio (Artículo 16), así: entre 1 y 20.000 habitantes, se pagará el 0,20\% de los ingresos brutos mensuales; entre 20.001 y 100.000 habitantes, el 0,40\% y más de 100.001 habitantes, el $4 \%$. Cuando los ingresos brutos mensuales sean superiores a 127 salarios mínimos legales vigentes en Colombia (equivalentes a \$111.480.981 de 2020, US33.458 americanos), el valor de la compensación será del $5,9 \%$, independientemente del número de habitantes. Esta disposición alivia la carga económica de estas televisiones, puesto que en la Resolución anterior debían pagar más dinero a la ANTV e, incluso, es más económica con respecto a lo previsto en el Acuerdo 009 de 2006, en el que se debía pagar el 1\% por cada canal codificado que se emitiera.

En producción propia, la Resolución 650 indica que se deben producir entre tres y seis horas semanales, dependiendo del número de asociados y no del tiempo de funcionamiento del canal comunitario, como lo establecía la Resolución 433. Lo que no cambia es el número de canales codificados que puede ofertar, siete, con lo que los sistemas comunitarios pierden competitividad frente a las TV por suscripción cuya oferta es ilimitada.

Con la Resolución 650, el gobierno Santos trata de recuperar el modelo de televisión comunitario para que cumpla con lo expresado en el punto 2.2.3 de los Acuerdos de La Habana del 2016:

En un escenario de fin del conflicto, los medios de comunicación comunitarios, institucionales y regionales, contribuirán al desarrollo y promoción de una cultura de participación, igualdad y no discriminación, convivencia pacífica, paz con justicia social y reconciliación, incorporando en sus contenidos valores no sexistas y de respeto al derecho de las mujeres a una vida libre de violencias (Oficina del Alto Comisionado para la Paz, 2016, p. 45).

\subsection{La televisión comunitaria en la presidencia de Iván Duque Márquez (2018-)}

La Ley 1978 de 2019, recientemente sancionada por el presidente Duque, delinea el futuro de la televisión, de la televisión comunitaria, así como de la radiodifusión en el país. Ciertamente, la norma crea las condiciones para que el capital privado nacional e internacional invierta en infraestructura de internet, de manera que este servicio llegue a las zonas rurales y alejadas, donde habita gran parte de la población pobre y vulnerable de Colombia (Artículo 1). Esto significa que el Gobierno Duque considera que el porvenir de la comunicación audiovisual está en la televisión por internet mediante la tecnología 5G, la cual multiplica las velocidades actuales de acceso y transmisión de la información.

La Ley permite que los operadores de televisión en Colombia, como la TV comunitaria y excepto la televisión pública, pasen de la modalidad de contrato por concesión (permiso, otorgamiento o derecho para el uso del espectro electromagnético por 10 años) a la de la adquisición de títulos habilitantes (permiso por 20 años), con lo cual se busca más seguridad jurídica para los inversores (Artículo 10). Aun así, todos ellos tendrán que pagar un 1,5\% de sus ingresos brutos anuales para la instalación, ampliación, modificación, operación y explotación de las redes provistas por el Estado con las que se fortalece el Fondo Único de las Tecnologías de la Información y la Comunicación y desde donde se destinan recursos para financiar los servicios de internet, radiodifusión sonora y TV pública. El documento advierte que en los últimos cuatro años la TV pública se ha desfinanciado en un $11,2 \%$, por lo que es necesario inyectarle más recursos para que sea sostenible. El permiso u otorgamiento del 
uso del espectro se podrá pagar hasta en un 60\% por medio de la construcción de infraestructura de internet en escuelas, bibliotecas, hospitales (Artículo 13).

La televisión comunitaria, según reza el documento El futuro digital es de todos (MinTic Colombia, 2018), será fundamental para avanzar en la provisión de redes y servicios de telecomunicaciones en todo el territorio nacional, para lo cual el Estado le brinda incentivos como los de eximirlas del pago por contraprestación del uso del espectro si poseen bandas de frecuencias para programas sociales del Estado que amplíen la cobertura en zonas rurales (Artículo 11). Independientemente de lo anterior, el sistema comunitario estará exento por cinco años del pago por contraprestación para el fortalecimiento del Fondo Único TIC, periodo en el que estos operadores podrán hacer inversiones en equipos para el suministro de internet (Artículo 36).

La Ley 1978 de 2019 liquida la Autoridad Nacional de Televisión (ANTV) y sólo deja funcionando la Comisión de Regulación de las Comunicaciones, única entidad que de forma autónoma se encarga de regular, vigilar y sancionar a los operadores y de garantizar la libre competencia. Otras funciones de la ANTV, como la de asignar el uso del espectro con base en estudios técnicos y económicos, son asumidas por el Ministerio de las TIC. La disposición no se refiere al número de horas propias, ni de canales codificados permitidos para la TV comunitaria.

\section{Discusión}

La Sentencia del Consejo de Estado del 2 de marzo de 2001 durante la presidencia de Pastrana (1998-2002), pudo haber incidido en el declive de las televisiones comunitarias y el soporte para que el Tratado de Libre Comercio entre Colombia y Estados Unidos le diera prioridad al capital privado de la televisión por suscripción. Tal disposición justifica la reducción del número de asociados, a sólo 6.000, para evitar que las televisiones comunitarias se vuelvan "monopolios" audiovisuales en las grandes y pequeñas ciudades, cuando tienen que competir con los grandes conglomerados de los medios por suscripción que cuentan con más recursos humanos, económicos, financieros y tecnológicos. De hecho, estos últimos en el acumulado de los últimos siete años el crecimiento ha sido del 37,8\% y en el 2019 llega a la suma de 5.942.158 suscriptores, con una penetración del $72 \%$ de los hogares colombianos. Sin embargo, el 91,3\% de los suscriptores han sido acaparados por cinco operadores, de los 59 que hay en el país: Claro (2.510.000), Directv (1.110.000), Tigo (1.180.000), Movistar (544.556) y ETB (113.708) (Periódico La República, 2019).

Tampoco resulta acertada la interpretación del Consejo de Estado según la cual se limita la cobertura de la televisión comunitaria para salvaguardar su propósito de "alcanzar fines, cívicos, recreativos, culturales o institucionales" porque este servicio podría extenderse con otros sectores que no tienen las mismas necesidades y objetivos (Consejo de Estado, 2001).

Paradójicamente, durante la presidencia de Uribe Vélez (2002-2010) se expide el Acuerdo 009 de 2006 con el que se desarrolla el servicio de televisión comunitaria, toda vez que se le permite tener 15.000 asociados, los pagos por compensación se limitan al $1 \%$ por cada canal codificado emitido, la producción propia depende del número de asociados y la CNTV intenta entregar incentivos a las buenas producciones comunitarias. Como consecuencia de estas disposiciones, la cantidad de TV comunitarias va en aumento hasta el año 2012, cuando se cuenta con 764 de estos medios de comunicación y 393.509 asociados. Es en este periodo presidencial, concretamente en el año 2007, cuando el Congreso de la República y el Ejecutivo aprueban el TLC que -como hemos visto- restringe la cobertura a 6.000 asociados. Vale aclarar, no obstante, que este acuerdo comercial sólo empieza aplicarse en el 2012.

En la presidencia de Santos (2010-2018), se expide la Resolución 433 de 2013 con la que se reglamenta ampliamente lo expresado en el TLC y prácticamente se crean las condiciones para que los sistemas comunitarios desaparezcan paulatinamente. Además de limitar a 6.000 sus asociados, la Resolución la obliga a producir más 
horas de producción propia de acuerdo con el tiempo que lleve funcionando la TV comunitaria (no por número de asociados) y a pagar un porcentaje por cada asociado con destino a financiar la televisión pública (no el $1 \%$ de los ingresos brutos por cada canal codificado, de los siete permitidos).

El resultado de lo expuesto en el TLC y la Resolución fue la reducción de un 52\% de estos sistemas y de su cantidad de asociados al pasar de 393.509 en el 2012 a 179.625 a julio de 2019 (caída acumulada del 54,3\%). Lo más seguro es que un grupo de asociados con capacidad adquisitiva haya sido captado por la TV por suscripción y el grupo de bajos ingresos económicos se haya quedado sin televisión cerrada. Adicionalmente, la desaparición de estos medios de proximidad pudo haber causado un detrimento patrimonial del Estado, puesto que cabe recordar que la televisión comunitaria, junto con la TV por suscripción, local con ánimo de lucro y privada de cobertura nacional, subsidia a la televisión pública mediante los aportes por compensación provenientes de sus ingresos brutos mensuales. Un argumento a favor de ello, es que la TV pública se ha desfinanciado en un 11,2\% desde el año 2015 (MinTic Colombia, 2018).

Aunque se podría pensar que las televisiones comunitarias que no aparecen en los registros de la ANTV transitaron hacia televisiones por suscripción o televisiones locales, los datos demuestran que no fue así pues estas dos últimas han reducido su número en los últimos años: Un 60\% de la TV local ha desaparecido desde el año 2015 hasta mediados de 2019, al pasar de 41 a 16, mientras que de las 64 TV por suscripción existentes en el 2013 sólo cuatro de ellas ya no funcionan. Lo más probable es que los sistemas comunitarios tal vez migraron a televisión digital por internet, con lo cual adquieren audiencias marginales y fragmentarias y están supeditadas a la calidad de la banda ancha de los municipios donde operan.

Es previsible que la disminución de televisiones locales y comunitarias esté propiciando un déficit de información local, como lo diagnosticó la Fundación para la Libertad de Prensa en el año de 2017. Pues bien, esta institución halló que, en 392 municipios colombianos, un 56,8\% de los 690 rastreados, no existía información o medios de comunicación locales por los que las personas tramitaran sus quejas, hicieran control político a los gobernantes o reprodujeran su cultura.

Otras consecuencias no menos trascendentales tienen que ver con que se impide que las comunidades organizadas desarrollen competencias para administrar un medio de comunicación, sin ánimo de lucro, pero sostenible económicamente y en el que estén representados distintos sectores sociales en su producción, gestión y control, así como para producir contenidos audiovisuales que busquen educar en la no discriminación, la igualdad, el servicio social y comunitario y que compitan contra contenidos comerciales dedicados, principalmente, al entretenimiento o al mantenimiento de hegemonías políticas, económicas y culturales.

El periodismo independiente se perjudica porque los reporteros pueden tener un sueldo digno, sin depender exclusivamente de la publicidad y los subsidios estatales, puesto que la gente paga por ver televisión y de esos recursos se pagan nóminas a grupos de periodistas, camarógrafos, editores para la producción de la programación local, así como a secretarias, instaladores que contribuyen al funcionamiento del medio de comunicación.

Los proveedores de contenidos de Estados Unidos también pierden con el TLC porque los canales codificados que ofertan como los Discovery, Fox, Espn, entre otros, sólo los pueden vender a un limitado mercado de televisiones por suscripción y a un mermado mercado de televisiones comunitarias.

Con la firma de los Acuerdos de Paz de La Habana, en los que se reivindica el papel de los medios comunitarios en el posconflicto, el presidente Santos trata de subsanar el error de la Resolución 433 de 2013, norma que le resta condiciones económicas a las TV comunitarias para funcionar y sostenerse. Para ello, promulga la Resolución 650 de 2018 con la que disminuye sus pagos por compensación, su producción propia de contenidos 
y deja abierta la posibilidad de que pueda contar con más de 6.000 asociados. Aun así, mantiene la misma cantidad de canales codificados que puede ofertar, siete, lo cual les impide competir en igualdad de oportunidades con la TV por suscripción.

En la actual presidencia de Duque Márquez (2018...), se expide la Ley 1978 de 2019 por medio de la cual se crean las condiciones para que el capital privado invierta en el mejoramiento del servicio de internet, medio por el que se concibe la televisión en el mediano y largo plazo. Llama la atención que el Gobierno se concentra en la televisión por internet, pese a que en el 2012 la anterior CNTV había actualizado el estándar de la televisión digital del DVB-T al DVB-T2 (El estándar DVB-T2 ofrece más capacidad para transportar canales con mejor definición y eventualmente contenidos en 3D, mejor sonido, economizar espectro y más interactividad), para propiciar el apagón analógico definitivo al finalizar el 2019.

Según Zavala, experto en televisión, cine, video, televisión y guionismo, el cambio obedece a que la TDT fue el modelo europeo promovido desde los años noventa, pero la transición tecnológica y la obsolescencia programada no ha funcionado bien en muchos países, como parece ser el caso colombiano. El autor agrega que:

La gente no quiere cambiar sus televisores viejos y la política pública detrás del modelo comercial ha fracasado parcialmente. Ahora que el consumo de televisión por internet superó al del cable y de los canales públicos de antena, es en un sentido natural girar hacia internet. Va a ser el vehículo de todas las demás tecnologías, así que la TDT no tiene mucho sentido ahora. No son incompatibles, pero es una tecnología que se volverá secundaria en muy poco tiempo, así que se ve como pérdida de dinero (Angulo, 2019).

En ese entorno, el consumo se ha inclinado por el de la televisión por internet, debido a la costumbre de usar el celular y los módems para ver programas de todo tipo por este sistema, por lo que el gobierno de Duque ve más útil invertir recursos en el mejoramiento de la infraestructura de conexión online. El problema radica en que la TDT era una de las formas de llegar con el servicio a regiones remotas y para que internet lo pueda hacer requiere de calidad y precios competitivos que beneficien a las personas de bajos ingresos. Si no se logra, puede generar marginación digital.

El papel de la televisión comunitaria parece ser el de llevar internet a zonas rurales, pues no tiene fundamento el que lo haga en ciudades donde existen operadores privados con abundantes recursos económicos, financieros y tecnológicos. Para ello, la Ley 1978 de 2019 ofrece incentivos como los de exonerarlas del pago de contraprestación del uso del espectro durante cinco años (Artículo 34) o en forma indefinida siempre que presten sus bandas de frecuencias para espacios sociales del Estado en sectores rurales (Artículo 11). Sin embargo, vale preguntarse hasta qué punto será rentable para un operador comunitario llevar televisión por internet en lugares remotos y si están en capacidad de competir con los conglomerados de medios que hayan acaparado este servicio.

Así las cosas, la discusión sobre cobertura no tiene sentido, pues cualquier persona puede observar los contenidos de la televisión comunitaria, pero sí se debe revisar el pago por compensación (que con la nueva Ley sería del 1,5\% de los ingresos brutos mensajes, más el permiso del uso del espectro por 20 años), el número de canales codificados que puede ofertar, la cantidad de horas de producción propia y, especialmente, si estos sistemas van a contar con capacitación de excelencia para la creación de contenidos transmedia en distintas plataformas.

Estas respuestas las tendrán que dar prontamente la Comisión de Regulación de las Comunicaciones y el Ministerio de las TIC, los entes a cuyo cargo queda la regulación del mercado de las telecomunicaciones y la entrega de las licencias de operación, respectivamente. 


\section{Conclusiones}

El Estado colombiano, quizás presionado por el capital privado de la televisión, ha venido restándole garantías para que la televisión comunitaria pueda funcionar y sostenerse económicamente. Así lo hemos evidenciado a partir del análisis de la normativa comercial (TLC entre Colombia y Estados Unidos) y audiovisual (acuerdo y resoluciones) y de las consecuencias de estas regulaciones: reducción de un 52\% de estas experiencias mediáticas y reducción de su número de asociados en un $54,3 \%$, los cuales pagan por ver el canal comunitario y canales codificados e incidentales (gratuitos). Es probable que algunos de ellos se hayan quedado sin televisión cerrada y otros, los de más ingresos, hayan sido cautivados por la televisión por suscripción, cuya lógica del crecer y crecer en una competencia que a todas luces es desigual está acabando con las televisiones comunitarias y con la oportunidad de que las comunidades organizadas crean medios de comunicación participativos y produzcan contenidos con vocación educativa y cultural en un país como Colombia donde el déficit de información local es del 56,8\% (Fundación para la Libertad de Prensa, 2017).

Si bien el Estado ha venido invirtiendo recursos para mejorar la infraestructura televisiva de manera que el servicio sea interactivo y en alta definición, como lo exigen estos tiempos, la eliminación paulatina de las televisiones comunitarias devuelve al país al precario estado comunicativo de los 70 en el Tercer Mundo, en momentos en que los promotores del Nuevo Orden Mundial de la Información (NOMIC) pedían justamente la descentralización de la información y se pronunciaban en contra de la concentración de los medios (Angulo, 2012).

En época de posconflicto, los medios comunitarios son importantes para promocionar la democracia, la no discriminación, la reivindicación social y la reconciliación (Oficina del Alto comisionado para la Paz, 2016), pero al desaparecer el modelo de televisión comunitaria lo más seguro es que el tránsito hacia una nación en paz pierda a un valioso aliado por el carácter popular y participativo que representa.

Finalmente, es necesario agilizar la aplicación de la Resolución 650 de 2018 -la cual reduce los costos de producción de contenidos y amplía la cobertura-, y los incentivos expuestos en la Ley 1978 de 2019, para que televisión comunitaria pueda funcionar, sostenerse y fortalecerse. Además, debe permitirle la oferta ilimitada de canales codificados para que pueda competir con las TV por suscripción.

\section{Referencias bibliográficas}

Angulo, L., Moreno, A. and Díaz, N. (2018) ‘Réquiem por la televisión comunitaria de Colombia : influencia de la legislación comercial y audiovisual del país en contra de esta televisión de proximidad', Revista lbérica de Sistemas e Tecnologias de Informação Iberian Journal of Information Systems and Technologies Recebido/Submission:, E 16, 11, pp. 234-246.

Angulo, S. (2012) 'Televisión y periodismo comunitarios en la ruta del desarrollo humano: modelos y fundamentos', p. 350. Available at: http://repository.ut.edu.co/bitstream/001/1547/1/RIUT-EBB-spa2015-Televisión y periodismo comunitarios en la ruta del desarrollo humano. Modelos y fundamentos.pdf.

Angulo, S. (2019) 'Entrevista a Diego Zavala Scherer’. Guadalajara: Maestría en Comunicación Digital Interactiva, p. 1.

ANTV (2019) Autoridad Nacional de Televisión ANTV, ANTV. Available at: https://www.antv.gov.co/index.php/informacion-sectorial/informacion-asociados-comunitarias (Accessed: 25 September 2019). 
Autoridad Nacional de Televisión (2006) 'Acuerdo 009 de 2006'. Available at: https://www.antv.gov.co/index.php/component/jdownloads/send/980-acuerdos-2006/844-acuerdo-004de-2006.

Autoridad Nacional de Televisión (2013) 'Resolucion 433 de 2013'. Bogotá. Available at: https://www.antv.gov.co/index.php/component/jdownloads/send/42-resoluciones-2013/1640resolucion-433-de-2013.

Autoridad Nacional de Televisión (2018) 'Resolucion 650 de 2018'. Bogotá. Available at: https://www.antv.gov.co/index.php/component/jdownloads/send/1235-proyectos-regulatorios2018/7123-resolucion-650-de-2018.

Autoridad Nacional de Televisión (2019) Directorios de televisión comunitaria de Colombia. Available at: https://www.antv.gov.co/index.php/component/jdownloads/category/16-directorio-operadores-tvcomunitaria?ltemid=-1 (Accessed: 6 September 2019).

Chaparro, M. (2002) Sorprendiendo al futuro. Comunicación para el desarrollo e información audiovisual. Primera ed. Barcelona: Los libros de la frontera (Comunicación). Barcelona: Los libros de la frontera (Comunicación).

Comisión Nacional de Telecomunicaciones de Venezuela (2002) 'Reglamento de Radiodifusion Sonora y Television Abierta comunitaria de Venezuela'. Available at: https://goo.gl/pvRKHU.

Consejo de Estado (2001) Sentencia 1999-05907 de marzo 2 DE 2001, Legis. Available at: http://legal.legis.com.co/document/Index?obra=jurcol\&document=jurcol_6f798a3f63964aabbf3ff180c8c0 ba42 (Accessed: 6 September 2019).

Dagron, A. G. (2019) TV comunitaria: ni púlpito, ni pulpo: pálpito. Available at: https://goo.gl/awA3AW (Accessed: 6 September 2019).

Fundación para la Libertad de Prensa (2017) El periodismo local se extingue en Colombia. Available at: https://flip.org.co/cartografias-informacion/ (Accessed: 6 September 2019).

García, María del Carmen y Berganza, M. R. (2005) 'El método científico aplicado a la investigación en Comunicación Mediática', in Berganza, María Rosa y Ruiz, J. A. (ed.) Investigar en comunicación. Guía práctica de métodos y técnicas de investigación social en comunicación. Madrid: McGraw Hill, pp. 19-42.

Gumucio Dagron, A. (2001) Haciendo olas: historias de comunicación participativa para el cambio social. New York.

Hernández, Roberto; Fernández, Carlos y Baptista, P. (2014) Metodología de la investigación. 6a. Edició. McGraw Hill. doi: 10.1192/bjp.112.483.211-a.

Jensen, K. B. (2014) 'La comunicación y los medios. Metodologías de investigación cualitativa y cuantitativa', in Jensen, K. B. (ed.) La comunicación y los medios. Metodologías de investigación cualitativa y cuantitativa. México D.F.: Fondo de Cultura Económica, pp. 385-410. doi: 10.1192/bjp.112.483.211-a.

Krohling, C. M. (2006) 'Televisión Comunitaria en Brasil : Antecedentes y participación popular en la gestión y en la programación', Redes.com, (3), pp. 347-368. Available at: https://goo.gl/EVZfbX.

Mincomercio de Colombia (2006) Tratado de Libre Comercio entre Colombia y Estados Unidos. Available at: http://www.fitac.net/noticias/documents/TextoTLC.pdf. 
MinTic Colombia (2018) El futuro digital es de todos. Bogotá. Available at:

http://micrositios.mintic.gov.co/modernizamos-sector-

tic/proyecto_ley_modernizacion_sector_tic_cartilla.pdf.

Neuendorf, K. (2002) The content analysis guidebook. Oaks: Sage.

Noboa Auz, M. L., Loor Saltos, M. G. and Del Pozo, V. H. (2018) 'Televisión Comunitaria: factores de éxito para el crecimiento en Ecuador', Killkana Social, 2(1), pp. 27-32. doi: 10.26871/killkana_social.v2i1.181.

Oficina del Alto comisionado para la Paz (2016) Acuerdos de Paz de La Habana, Acuerdo final para la terminación eel conflicto y la construcción de una paz estable $Y$ duradera. Available at: http://www.altocomisionadoparalapaz.gov.co/procesos-y-conversaciones/Documentos compartidos/2411-2016NuevoAcuerdoFinal.pdf.

Parra, J. D. (2006) 'Palabra Clave’, Palabra Clave, 9(2), pp. 67-76.

Periódico La República (2019) Televisión por suscripción creció 20\% en los últimos cinco años en Colombia. Available at: https://www.larepublica.co/empresas/television-por-suscripcion-crecio-20-en-los-ultimoscinco-anos-en-colombia-2887731 (Accessed: 9 October 2019).

Presidencia de la República (2019) Ley 1978 de 2019. Available at: https://dapre.presidencia.gov.co/normativa/normativa/LEY 1978 DEL 25 DE JULIO DE 2019.pdf (Accessed: 6 September 2019).

Rodríguez, E. (1998) ‘Algunas anotaciones para una posible historia de la televisión local y comunitaria en Colombia', Señales de Humo, 1, pp. 13-22.

Toro, Iván Darío y Parra, R. D. (2010) Fundamentos epistemológicos de la investigación y la metodología de la investigación. Cuantitativa/cualitativa. Bogotá: Fondo Editorial Universidad Eafit.

Toro Bravo, M. J. P., Mullo Lopez, M. A. and Hinojosa Becerra, P. D. M. (2019) 'La televisión comunitaria en la región central de Ecuador, TV MICC y PURUHÁ TV', Chasqui. Revista Latinoamericana de Comunicación, (140), pp. 215-230. doi: 10.16921/chasqui.v0i140.3929.

Villarrubia- Martínez, A., Aguaded-Gómez, I. and Delgado-Ponce, Á. (2019) ‘Implementación de la Televisión digital en Chile: ¿una oportunidad real para la TV Comunitaria?', Chasqui. Revista Latinoamericana de Comunicación, (140), pp. 279-296. doi: 10.16921/chasqui.v0i140.3878.

\section{(cc) $\mathrm{EY}$-NC}

Esta obra está bajo una Licencia Creative Commons Atribución-NoComercial 4.0 Internacional 